PROCEEDINGS OF THE AMERICAN MATHEMATICAL SOCIETY

Volume 126, Number 3, March 1998, Pages 917-924

S 0002-9939(98)04158-6

\title{
THE 6-PROPERTY FOR SIMPLICIAL COMPLEXES AND A COMBINATORIAL CARTAN-HADAMARD THEOREM FOR MANIFOLDS
}

\author{
J. M. CORSON AND B. TRACE
}

(Communicated by Ronald A. Fintushel)

\begin{abstract}
The 6-property for 2-dimensional simplicial complexes is the condition that every nontrivial circuit in the link of a vertex has length greater than or equal to six. If a compact $n$-manifold $M$ has a 2 -dimensional spine with the 6-property, then we show that the interior of $M$ is covered by euclidean $n$-space. In dimension $n=3$, we show further that such a 3-manifold is Haken.
\end{abstract}

\section{INTRODUCTION}

It is a classical result (the Cartan-Hadamard Theorem) that if $M$ is a complete $n$-dimensional Riemannian manifold, without boundary, with nonpositive sectional curvature, then the universal cover of $M$ is diffeomorphic to $\mathbb{R}^{n}$. Our aim here is to prove an analogue of this theorem, for bounded manifolds, where the geometric hypothesis on the manifold is replaced by a combinatorial nonpositive curvature condition on a spine of the manifold.

The combinatorial versions of nonpositive curvature we have in mind are related to regular tessellations of euclidean space. Confining our attention, as we shall do throughout, to 2-dimensional spines, there are three such conditions corresponding to the three regular tessellations of the plane. (Recall that it is possible to subdivide the plane into regular $p$-gons such that each vertex is $q$-valent if and only if $(p, q)$ is one of the pairs $(3,6),(4,4)$, or $(6,3)$.)

To simplify the exposition, we concentrate only on the first condition; the other two cases can be handled similarly. To be precise, we consider compact manifolds supporting triangulations that collapse onto a 2-dimensional subcomplex (spine) with the 6-property, meaning that the link (in the spine) of each vertex is a graph of girth at least 6 . (Recall that the girth of a graph is the minimum number of edges in a circuit.) See $[\mathrm{CT}]$ for more details on the 6 -property. Our main result is

Theorem 4.1. Suppose $M^{n}$ is a compact $n$-manifold that has a 2-dimensional spine with the 6-property. Then the interior of the universal cover of $M^{n}$ is (topologically) homeomorphic to $\mathbb{R}^{n}$.

Received by the editors March 26, 1996 and, in revised form, September 3, 1996.

1991 Mathematics Subject Classification. Primary 57M20, 57N10, 20 F06.

Key words and phrases. Manifold, spine, universal cover, 6-property, collapsing.

(C)1998 American Mathematical Society 
Given any finite 2-dimensional simplicial complex $K$ with (or without) the 6property, it is well-known that $K$ is a spine of some compact (PL) $n$-manifold, for any integer $n \geq 4$. It is an interesting question as to whether or not $K$ is a spine of some compact 3-manifold. An even more interesting question is: which compact 3manifolds have 2 -dimensional spines with the 6-property? As a necessary condition, we find (Theorem 4.2) that such a 3-manifold must be Haken. At this time, we do not know whether the converse is true, though it seems unlikely. Many examples of 3-manifolds with spines of this type can be found among the knot complements. However, there are knots, for example doubles of fibered knots, for which we do not know whether the complement has a spine with the 6 -property.

With regard to the first question, we remark that in $[\mathrm{CT}]$ we give a necessary and sufficient condition for a finite 2-dimensional simplicial complex $K$ to embed into an orientable 3 -manifold, in which case $K$ would be a spine of a compact orientable 3-manifold.

For use in establishing the main theorem, and of independent interest, we introduce three, length non-increasing, elementary operations on edge-paths in a simplicial complex. Then we show (Theorem 3.1) that if $K$ is a 2-dimensional simplicial complex with the 6-property, we can (essentially) pass from one edge-path to any homotopic edge-path via a finite sequence of these operations. This should be compared with the notion, in theoretical computer science, of a confluent presentation of a monoid.

Connection with recent developments in dimension three. In all known examples of compact aspherical 3-manifolds $M$, the interior of $M$ is covered by euclidean space $\mathbb{R}^{3}$ (it is unknown whether this is always the case). The special position of dimension three, in this regard, should be noted: In dimensions less than three, every compact aspherical manifold is such that the universal cover of its interior is euclidean space. On the other hand, nothing like this is possible in dimensions greater than three. In particular, there are examples, first discovered by M. Davis [D], of closed aspherical $n$-manifolds whose universal cover is not euclidean $n$-space, for each $n \geq 4$.

Recently, V. Poénaru $[\mathrm{P}]$ and A. Casson (see $[\mathrm{SG}]$ ) have discovered new conditions under which certain 3-manifolds are covered by $\mathbb{R}^{3}$. Their conditions have been abstracted by Brick and Mihalik [BM] (also see [S]) into the following grouptheoretical property. A finitely presented group $G$ is said to be quasi simply filtrated (abbreviated $q s f$ ) if for some (then it turns out to be true for any) compact, connected polyhedron $P$ with fundamental group $G$, the universal cover $\widetilde{P}$ has the property: for each compact subset $X$ of $\widetilde{P}$, there exists a compact, 1-connected polyhedron $A$ and a PL map $f: A \rightarrow \widetilde{P}$ that maps $f^{-1}(X)$ homeomorphically onto $X$.

Theorem (Casson, Poénaru, Brick-Mihalik, Stallings). Let $M$ be an irreducible, compact, aspherical 3-manifold. If $\pi_{1}(M)$ is qsf, then the universal cover of the interior of $M$ is homeomorphic to $\mathbb{R}^{3}$.

It is not known whether or not all compact 3-manifolds have qsf fundamental groups. And a positive solution to the Poincaré conjecture would imply that all aspherical 3-manifolds are automatically irreducible.

We show that the fundamental group of a finite, connected, 2-dimensional simplicial complex with the 6-property is qsf in a very strong sense. Namely, by 
Theorem 3.5, its universal cover is a monotone union of a sequence of collapsible subcomplexes. Our main theorem (Theorem 4.1) then follows quickly, by appealing to M. Brown's theorem about a monotone union of $n$-cells; see $\S 4$.

It should be noted that it follows from work of Alonso and Bridson [AB] that the fundamental group of a finite, connected, 2-dimensional simplicial complex with the 6-property is semihyperbolic, hence qsf by a result of Mihalik and Tschantz [MT]. Thus, in dimension three, the merit of our approach is that we do not need the irreducibility assumption. Moreover, it follows from our proof of Theorem 4.1 that all compact 3-manifolds that have 2-dimensional spines with the 6-property are irreducible, indeed Haken (Theorem 4.2).

\section{Preliminaries}

Unless stated to the contrary, we work in the PL category. Let $K$ be a simplicial complex. By a directed edge of $K$ we mean an oriented 1-simplex $e=\left[v_{0}, v_{1}\right]$. The vertex $v_{0}$ is called the origin of $e$, denoted $o(e)$, and the vertex $v_{1}$ is called the terminus of $e$, denoted $t(e)$. We put $\bar{e}=\left[v_{1}, v_{0}\right]$, called the inverse of $e$. An edge-path in $K$ is a finite sequence $\alpha=e_{1} e_{2} \cdots e_{n}$ of directed edges in $K$ such that $t\left(e_{i}\right)=o\left(e_{i+1}\right)$ for $1 \leq i \leq n-1$. The inverse of $\alpha$ is the edge-path $\alpha^{-1}=\bar{e}_{n} \cdots \bar{e}_{1}$.

Given an edge-path $\alpha=e_{1} \cdots e_{n}$ (of length $n$ ), denote by $\phi_{\alpha}:[0,1] \rightarrow|K|$ the simplicial map where $[0,1]$ is triangulated so that $\left\{0, \frac{1}{n}, \frac{2}{n}, \ldots, 1\right\}$ is the vertex set and $\phi_{\alpha}\left(\frac{i}{n}\right)=t\left(e_{i}\right)=o\left(e_{i+1}\right)$. We say that edge-paths $\alpha$ and $\beta$ are path-homotopic if $\phi_{\alpha}$ and $\phi_{\beta}$ are homotopic (rel $\{0,1\}$ ) in $|K|$. It is well-known that concatenation of edge-paths determines a product making the set of path-homotopy classes into a groupoid, and the mapping $\alpha \mapsto \phi_{\alpha}$ induces an isomorphism with the fundamental groupoid of $|K|$.

Suppose $\alpha$ is a closed edge-path with endpoints at the vertex $v_{0}$. Then it is well-known that $\alpha$ is path-homotopic to the trivial path at $v_{0}$, null-homotopic for short, if and only if there exists a van Kampen diagram for $\alpha$, that is, a pair $(D, \phi)$ where $D$ is a finite, 1-connected 2-complex embedded in the plane and $\phi: D \rightarrow|K|$ is a combinatorial map such that a boundary cycle is labeled by $\alpha$. Furthermore, if we choose a van Kampen diagram of least area, meaning number of 2-cells in $D$, then the diagram will be reduced in the sense that the induced map $\operatorname{lk}(v, D) \rightarrow$ $\operatorname{lk}(f(v), K)$ is an immersion for each vertex $v$ of $D$; see, for example, [LS]. In particular, if $K$ has the 6-property and $(D, \phi)$ is a reduced van Kampen diagram in $K$, then $D$ will also have the 6-property. By the area of a null-homotopic edge-path $\alpha$, we mean the least area of a van Kampen diagram for $\alpha$.

The following special case of Lyndon's curvature formula is employed in the next section; a proof is included for completeness. First we explain the terminology. By a combinatorial 2-disc we mean a combinatorial 2-complex, homeomorphic to a 2 -disc. The length of the attaching path for a 2-cell $\sigma$ is called its degree, denoted $d(\sigma)$; and the number of edges incident with a given vertex $v$ is called the degree or valence of $v$, denoted $d(v)$.

Lemma 2.1. Let $D$ be a combinatorial 2-disc such that each 2-cell has degree 3 and each interior vertex has degree at least 6 . Then

$$
\sum_{\partial D}[4-d(v)] \geq 6
$$

summing over the boundary vertices of $D$. 


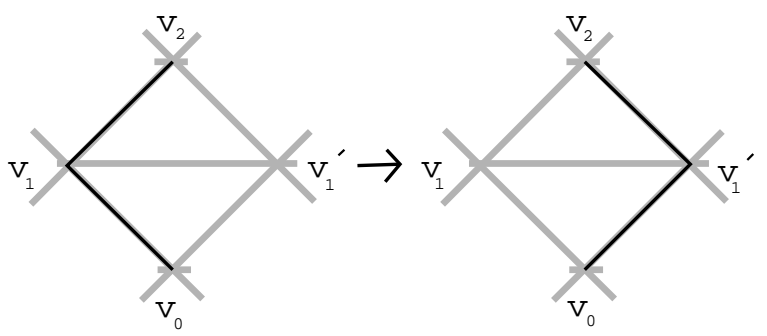

FiguRE 1. Elementary exchange

Proof. Denote by $V, E$, and $F$ the number of vertices, 1-cells, and 2-cells of $D$, respectively. Similarly, denote by $V_{\infty}$ and $E_{\infty}$ the number of vertices and 1-cells in $\partial D$, respectively. Then

$$
2 E=\sum d(v) \geq 6\left(V-V_{\infty}\right)+\sum_{\partial D} d(v)=6 V-2 V_{\infty}-\sum_{\partial D}[4-d(v)] .
$$

But $3 F=2 E-E_{\infty}=2 E-V_{\infty}$, as 2-cells have degree 3 and $\partial D$ is a circle. Whence

$$
\sum_{\partial D}[4-d(v)] \geq 6(V-E+F)=6,
$$

as the Euler characteristic of $D$ is 1 .

\section{Elementary OPERATIONS ON EDGE-PATHS}

Let $K$ be a simplicial complex. We introduce three elementary operations on edge-paths in $K$.

1. (Free reduction): If $\alpha$ is an edge-path containing a subpath of the form $e \bar{e}$ and $\beta$ is the edge-path obtained by deleting this subpath, then we call the passage from $\alpha$ to $\beta$ a free reduction.

2. (Short-cut): By a short-cut we mean the passage from one edge-path to another by replacing an occurrence of a path $\left[v_{0}, v_{1}\right]\left[v_{1}, v_{2}\right]$ by the single edge $\left[v_{0}, v_{2}\right]$, where $v_{0}, v_{1}, v_{2}$ are vertices of a 2 -simplex in $K$.

3. (Elementary exchange): The passage from one edge-path to another, obtained by replacing a subpath $\left[v_{0}, v_{1}\right]\left[v_{1}, v_{2}\right]$ by $\left[v_{0}, v_{1}^{\prime}\right]\left[v_{1}^{\prime}, v_{2}\right]$, is termed an elementary exchange, if $\tau=\left\{v_{1}, v_{1}^{\prime}\right\}$ is a 1-simplex such that $\tau \cup\left\{v_{0}\right\}$ and $\tau \cup\left\{v_{2}\right\}$ are distinct 2-simplices; see Figure 1.

Note that none of the elementary operations alter the endpoints of an edge-path. Free reductions and short-cuts reduce the length of an edge-path, whereas elementary exchanges preserve length. Elementary exchanges are invertible operations, but free reductions and short-cuts are not.

Definition. We say that two edge-paths $\alpha$ and $\beta$ are exchangeable, and write $\alpha \equiv \beta$, if it is possible to pass from $\alpha$ to $\beta$ via a finite sequence of elementary exchanges. By saying that an edge-path $\alpha$ is strongly reduced, we mean that for each edge-path $\beta$, exchangeable with $\alpha, \beta$ does not admit a free reduction or shortcut.

It is clear that $\equiv$ is an equivalence relation on the set of edge-paths of $K$, and exchangeable edge-paths are path-homotopic. Indeed, if there is a finite sequence of elementary operations passing from an edge-path $\alpha$ to an edge-path $\beta$, then $\alpha$ 
and $\beta$ are path-homotopic. The next theorem can be viewed as a converse, of sorts, for 2 -complexes with the 6 -property.

Theorem 3.1. Suppose that $K$ is a 2-dimensional simplicial complex with the 6 property, and let $\beta$ be a strongly reduced edge-path in $K$. If $\alpha$ is any edge-path, path-homotopic to $\beta$, then there is a finite sequence of free reductions, short-cuts, and elementary exchanges passing from $\alpha$ to $\beta$.

Proof. By initially applying elementary operations to $\alpha$, we may assume that $\alpha$ is also strongly reduced. Thus, it suffices to show that if $\alpha$ and $\beta$ are two strongly reduced edge-paths that are path-homotopic, then $\alpha \equiv \beta$. This we prove by induction on the area of the null-homotopic edge-path $\alpha \beta^{-1}$.

To this end, let $(D, \phi)$ be a minimal area van Kampen diagram for $\alpha \beta^{-1}$. Such a diagram is reduced; thus each interior vertex of $D$ has valence greater than or equal to 6 . By taking subpaths if necessary, we may assume that $\alpha$ and $\beta$ do not have a nontrivial common initial or terminal segment. Then, since $\alpha$ and $\beta$ are strongly reduced, no vertex of $D$ has valence one.

Choose an extremal disc $D_{0}$ of $D$, that is, a properly embedded subcomplex, homeomorphic to a 2-disc, whose complement in $D$ is connected; see [LS, p. 247]. Note that there are vertices $v_{1}$ and $v_{2}$ (not necessarily distinct) in the boundary of $D_{0}$ such that the two boundary segments joining them are labeled by subpaths $\alpha_{0}$ and $\beta_{0}$ (one of which could be trivial) of $\alpha$ and $\beta$, respectively. By Lemma 2.1, it follows that at least three boundary vertices of $D_{0}$ have degree less than 4 . (Every vertex has degree at least 2.) Hence, there is a vertex $v$, not equal to $v_{1}$ or $v_{2}$, in the boundary of $D_{0}$ such that $d(v)$ is either 2 or 3 . It follows that the star in $D$ of the vertex $v$ contains only one or two 2-cells.

Deleting the open star in $D$ of the vertex $v$ corresponds to performing either a short-cut or an elementary exchange on one of the edge-paths $\alpha$ or $\beta$. Since $\alpha$ and $\beta$ are strongly reduced, a short-cut is impossible. Thus, there is an elementary exchange passing from, say $\alpha$, to an edge-path $\gamma$ and the area of $\gamma \beta^{-1}$ is (at least) two less than that of $\alpha \beta^{-1}$. Thus, $\alpha \equiv \gamma \equiv \beta$ by the induction hypothesis.

Remark 3.2. Let $K$ be a finite 2 -dimensional simplicial complex with the 6 -property. Then the theorem provides an algorithm deciding whether a given closed edge-path $\alpha$ is null-homotopic, roughly as follows. List the finitely many edge-paths that can be obtained from $\alpha$ by an elementary operation. Then form a second list consisting of all the edge-paths that can be obtained from some edge-path in the first list by an elementary operation. Continuing in this fashion, eventually one of two possibilities will occur: either the trivial path will be in the list, in which case $\alpha$ is null-homotopic, or every edge-path in the list will have already occurred in a previous list. In the later case, it follows that no sequence of elementary operations leads from $\alpha$ to a trivial path, and hence, $\alpha$ is essential by the theorem.

It should be noted that there is an alternative, if not equivalent, algorithm employing Lyndon's isoperimetric inequality [LS, Theorem V.6.2].

Given directed edges $e$ and $e^{\prime}$ in a simplicial complex $K$ such that $o(e)=o\left(e^{\prime}\right)=$ $v$, let $\rho\left(e, e^{\prime}\right)$ denote the length of a shortest path in $\operatorname{lk}(v, K)$ joining $t(e)$ and $t\left(e^{\prime}\right)$, letting $\rho\left(e, e^{\prime}\right)=\infty$ if no such path exists. We say that an edge-path $e_{1} \cdots e_{n}$ is locally geodesic if $\rho\left(\bar{e}_{i}, e_{i+1}\right) \geq 3$ for all $1 \leq i<n$. Observe that a locally geodesic edge-path does not permit any elementary operations. Consequently, if $K$ has the 6 -property, a closed, locally geodesic edge-path in $K$ is essential by Theorem 3.1. 
Remark 3.3. Suppose $K$ is a connected, 2-dimensional simplicial complex with the 6-property. Assume further that $K$ is closed, by which we mean $K$ is finite and every 0 - and 1 -simplex is a proper face of at least two simplices; i.e., $K$ is finite and does not admit any elementary collapses. Then, by choosing edges in succession, so as to construct a locally geodesic edge-path in $K$, we eventually obtain the situation where some subpath is closed (and locally geodesic), as $K$ is finite. This subpath being essential, it follows that $\pi_{1}(K)$ is nontrivial. In fact, more is true: Unless $|K|$ is homeomorphic to the torus or Klein bottle, $\pi_{1}(K)$ contains a nonabelian free subgroup; see $[\mathrm{CT}]$.

Our main application of Theorem 3.1 is in showing that locally finite, 1-connected 2 -complexes with the 6 -property have a rather simple combinatorial structure. First we need a lemma.

Lemma 3.4. Let $K$ be a finite, 1-connected, 2-dimensional simplicial complex with the 6-property. Then $K$ is collapsible.

(We have been informed that S. Gersten has proven a more general version of Lemma 3.4, unpublished.)

Proof. Let $L$ be a connected subcomplex of $K$ that does not admit an elementary collapse, $L$ having more than one vertex. Then either $L$ is 1-dimensional and $\pi_{1}(L)$ is a nontrivial free group, or $L$ is 2-dimensional and every 0- and 1-simplex of $L$ is a proper face of at least two simplices of $L$. In the latter case, since $L$ inherits the 6-property, $\pi_{1}(L)$ is also nontrivial as remarked above. It follows that any 1connected subcomplex of $K$ containing more than one vertex admits an elementary collapse. Hence, any random sequence of elementary collapses, starting with $K$, eventually terminates in a one-point subcomplex.

Theorem 3.5. Let $K$ be a locally finite, 1-connected, 2-dimensional simplicial complex with the 6-property. Then $K$ is a monotone union of a sequence of collapsible subcomplexes.

Proof. Fix a vertex $v_{0}$ in $K$. For each integer $n$, let $L_{n}$ be the full subcomplex of $K$ generated by the vertices that can be joined to $v_{0}$ by an edge-path of length at most $n$. Note that $K=\bigcup_{n=1}^{\infty} L_{n}$, a monotone union of finite, connected subcomplexes. Hence, the theorem follows from the lemma once we verify that $\left|L_{n}\right|$ is simply connected for each $n$.

For this, let $\alpha=e_{1} \cdots e_{n}$ be a closed edge-path in $L_{n}$ with endpoints at $v_{0}$. Put $v_{i}=t\left(e_{i}\right)=o\left(e_{i+1}\right)$. For each $i$, let $\gamma_{i}$ be an edge-path from $v_{0}$ to $v_{i}$ of minimal length. Then

$$
\gamma=e_{1} \gamma_{1}^{-1} \gamma_{1} e_{2} \gamma_{2}^{-1} \gamma_{2} \cdots e_{n-1} \gamma_{n-1}^{-1} \gamma_{n-1} e_{n}
$$

is an edge-path in $L_{n}$ that freely reduces to $\alpha$.

Since $|K|$ is simply connected, for each $i$ there is a finite sequence of elementary operations passing from $\gamma_{i} e_{i+1}$ to $\gamma_{i+1}$, by Theorem 3.1. Each edge-path in this sequence clearly lies in $L_{n}$. Thus, $\gamma_{i} e_{i+1}$ and $\gamma_{i+1}$ are path-homotopic in $L_{n}$. We conclude that $\gamma$, and hence $\alpha$, is null-homotopic in $L_{n}$, as required.

\section{Manifolds With 6-PRoperty SPINES}

We say that a simplicial complex $K$ is a spine of an $n$-manifold $M^{n}$ if $M^{n}$ supports a triangulation that collapses to a subcomplex isomorphic to $K$. For a 
general reference on piecewise linear topology, we refer the reader to the book by Rourke and Sanderson [RS].

In this section we consider compact manifolds that have 2-dimensional spines enjoying the 6-property. First of all, it should be noted that 2-dimensional simplicial complexes with the 6-property are aspherical. This follows from the well-known fact that the 2 -sphere $S^{2}$ does not support a triangulation with the 6-property. Hence, the manifolds we shall be considering are aspherical with fundamental groups of cohomological dimension two. Throughout, all manifolds are assumed to be connected.

Theorem 4.1. Suppose $M^{n}$ is a compact $n$-manifold that has a 2-dimensional spine with the 6-property. Then the interior of the universal cover of $M^{n}$ is (topologically) homeomorphic to $\mathbb{R}^{n}$.

Proof. Invoking Theorem 3.5, we see that there is a triangulation of the universal cover $\widetilde{M}^{n}$ which is a monotone union of a sequence of regular neighborhoods of collapsible subcomplexes. Since a regular neighborhood (in a manifold) of a collapsible subcomplex is an $n$-cell, we have that $\operatorname{Int} \widetilde{M}^{n}$ is a monotone union of open $n$-cells. The result now follows from M. Brown's Theorem [B].

More can be said in dimension three. Suppose $M^{3}$ is a 3 -manifold with a 2dimensional spine $K$ enjoying the 6-property. Any (PL-) embedding of $S^{2}$ into Int $M^{3}$ lifts to the universal cover where it bounds a 3 -cell-as the universal cover is a monotone union of PL 3 -cells and $S^{2}$ is compact. Since the covering projection embeds this ball into $M^{3}$, it follows that $M^{3}$ is irreducible. Furthermore, being aspherical, it follows that $M^{3}$ does not admit a two-sided (PL-) embedding of the projective plane $\mathbb{P}^{2}$. Therefore, $M^{3}$ is $\mathbb{P}^{2}$-irreducible.

Since it is well-known that compact, $\mathbb{P}^{2}$-irreducible 3 -manifolds with non-empty boundary are Haken, we have:

Theorem 4.2. Suppose $M^{3}$ is a compact 3-manifold that has a 2-dimensional spine with the 6-property. Then $M^{3}$ is Haken.

\section{REFERENCES}

[AB] J. M. Alonso and M. R. Bridson, Semihyperbolic groups, Proc. London Math. Soc. (3) 70 (1995), 56-114. MR 95j:20033

[BM] S. G. Brick and M. L. Mihalik, The qsf property for groups and spaces, Math. Z. 220 (1995), 207-217. MR 96i:57009

[B] M. Brown, The monotone union of open $n$-cells is an open n-cell, Proc. Amer. Math. Soc. 12 (1961), 812-814. MR 23:A4129

[CT] J. Corson and B. Trace, Geometry and algebra of nonspherical 2-complexes, J. London Math. Soc. 54 (1996), 180-198. CMP 96:14

[D] M. W. Davis, Groups generated by reflections and aspherical manifolds not covered by Euclidean space, Ann. of Math. 117 (1983), 293-324. MR 86d:57025

[LS] R. C. Lyndon and P. E. Schupp, Combinatorial group theory, Ergeb. Math., Bd. 89, Springer, New York, 1977. MR 58:28182

[MT] M. L. Mihalik and S. T. Tschantz, Tame combings of groups, Trans. Amer. Math. Soc. (to appear). CMP 96:12

[P] V. Poénaru, Almost convex groups, Lipschitz combing, and $\pi_{1}^{\infty}$ for universal covering spaces of closed 3-manifolds, J. Differential Geom. 35 (1992), 103-130. MR 93d:57032

[RS] C. P. Rourke and B. J. Sanderson, Introduction to piecewise-linear topology, Ergeb. Math., Bd. 69, Springer, New York, 1972. MR 50:3236 
[S] J. R. Stallings, Brick's quasi simple filtrations for groups and 3-manifolds, Geometric group theory (G. A. Niblo and M. A. Roller, eds.), vol. 1, Cambridge University Press, Cambridge, 1993, pp. 188-203. MR 94k:57004

[SG] J. Stallings and S. M. Gersten, Casson's idea about 3-manifolds whose universal cover is $\mathbb{R}^{3}$, Internat. J. Algebra Comput. 1 (1991), 395-406. MR 93b:57018

Department of Mathematics, University of Alabama, Box 870350, Tuscaloosa, AlABAMA 35487-0350

E-mail address: jcorson@mathdept.as.ua.edu

E-mail address: btrace@mathdept.as.ua.edu 